\title{
SMM: An R Package for Estimation and Simulation of Discrete-time semi-Markov Models
}

\author{
by Vlad Stefan Barbu, Caroline Bérard, Dominique Cellier, Mathilde Sautreuil and Nicolas Vergne
}

\begin{abstract}
Semi-Markov models, independently introduced by Lévy (1954), Smith (1955) and Takacs (1954), are a generalization of the well-known Markov models. For semi-Markov models, sojourn times can be arbitrarily distributed, while sojourn times of Markov models are constrained to be exponentially distributed (in continuous time) or geometrically distributed (in discrete time). The aim of this paper is to present the R package SMM, devoted to the simulation and estimation of discretetime multi-state semi-Markov and Markov models. For the semi-Markov case we have considered: parametric and non-parametric estimation; with and without censoring at the beginning and/or at the end of sample paths; one or several independent sample paths. Several discrete-time distributions are considered for the parametric estimation of sojourn time distributions of semi-Markov chains: Uniform, Geometric, Poisson, Discrete Weibull and Binomial Negative.
\end{abstract}

\section{Introduction}

Semi-Markov models, independently introduced by Lévy (1954), Smith (1955) and Takacs (1954), are a generalization of the well-known Markov models. For semi-Markov models, sojourn times can be arbitrarily distributed, while sojourn times of Markov models are constrained to be exponentially distributed (in continuous time) or geometrically distributed (in discrete time). For this reason, semiMarkov processes are more general and more adapted for applications than the Markov processes.

Semi-Markov processes have become important tools in probability and statistical modeling with applications in various domains like survival analysis, biology, reliability, DNA analysis, insurance and finance, earthquake modeling, meteorology studies, etc.; see, e.g., Heutte and Huber-Carol (2002), Ouhbi and Limnios (2003), Chryssaphinou et al. (2008), Janssen and Manca (2006), Votsi et al. (2012), D'Amico et al. (2013), Votsi et al. (2014), D’ Amico et al. (2016b), Barbu et al. (2016), D'Amico et al. (2016a) for semi-Markov processes in continuous or discrete time with various applications and Sansom and Thomson (2001), Bulla and Bulla (2006), Barbu and Limnios (2008), for hidden semi-Markov models and applications in climatology, finance and DNA analysis.

Note that the semi-Markov theory is developed mainly in a continuous-time setting, while utterly less works address the discrete-time case. We refer the reader to Limnios and Oprisan (2001) for continuous-time framework and to Barbu and Limnios (2008) and references therein for discrete-time framework. The R package SMM that we present in this paper is developed in discrete time. Note that undertaking works also in discrete time (modeling stochastic tools, associated estimation procedures, corresponding software, etc.) is an important issue for several reasons. In our opinion, the most relevant of these reasons is that the time scale is intrinsically discrete in several applications. For instance, in DNA studies, when modeling a nucleotide or protein sequence by means of a stochastic process, the "time" of that process is in fact the position along the sequence, so it is discrete. Other examples can be found in some reliability/survival analysis applications where the time represents the number of cycles of a system or the counting of days/hours/etc. We can argue further for the importance of developing works also in discrete time, in parallel to their analogous ones developed in continuous time. For instance, we can mention the simplicity of computations in discrete time, the fact that a discrete-time stochastic process does not explode, the potential use of discrete processes after the discretization of continuous ones, etc.

Few $\mathrm{R}$ packages have been developed to handle semi-Markov models or hidden semi-Markov models. For semi-Markov models we have the recent semiMarkov R package (Król and Saint-Pierre, 2015) that performs maximum likelihood estimation for parametric continuous-time semi-Markov processes, where the distribution can be chosen between Exponential, Weibull or exponentiated Weibull. That package computes associated hazard rates; covariates can also be taken into account through the Cox proportional hazard model. Two R packages are also dedicated to hidden semiMarkov models, implementing estimation and prediction methods: the hsmm R package (Bulla et al., 2010) and the mhsmm R package ( $\mathrm{O}^{\prime}$ Connell and Højsgaard, 2011).

Note that there is no R package developed for discrete-time multi-state semi-Markov models. Thus the purpose of this paper is to present an R package that we have developed, called SMM, which performs parametric and non-parametric estimation and simulation for multi-state discrete-time semi-Markov processes. For the parametric estimation, several discrete distributions are considered 
for the sojourn times: Uniform, Geometric, Poisson, Discrete Weibull and Negative Binomial. The non-parametric estimation concerns the sojourn time distributions, where no assumptions are made on the shape of distributions. Moreover, the estimation can be done on the basis of one or several trajectories, with or without censoring. The aim of this paper is to describe the different possibilities of this package. To summarize, the package SMM that we present deals with different problems:

- Parametric estimation for sojourn time distributions (Uniform, Geometric, Poisson, Discrete Weibull and Negative Binomial) or non-parametric estimation;

- One or several sample paths;

- Four different types of sojourn times: a general one depending on the current state and on the next state to be visited, one depending only on the next state, one depending only on the current state, and one depending neither on the current state nor on the next state;

- Four different types of censoring: censoring at the beginning of sample paths, censoring at the end of sample paths, censoring at the beginning and at the end of sample paths or no censoring at all.

Let us make some remarks about these points.

First, concerning the censoring, the simplest situation is the one when all the sojourn times are completely observed (non censored). A more complicated and realistic framework is when the last sojourn time is not completely observed, thus being right censored; in most practical situations this case occurs. An analogous situation is when the first sojourn time is not completely observed, thus being also right censored. In practice, this last case occurs when one does not know the beginning of a phenomenon modeled by a semi-Markov chain. Note that this censoring at the beginning of the sample path is a right censoring (not a left censoring); indeed, when the first sojourn time is censored as in our paper, the available information is that the real sojourn time (that is not observed) is greater than this censored observed time. Thus we are clearly in a right censoring framework, although this happens at the beginning (i.e., left) of the trajectory, which could seem confusing. The most complete framework is when both the first and the last sojourn times are right censored.

Second, when considering estimation starting from several independent sample paths of a semiMarkov chain, it is assumed that all the trajectories are censored in the same way; note that this is not a real constraint, but we imposed this condition only in order to avoid useless technical notations that would make the comprehension more difficult.

Third, note that it is important for the four types of models (of sojourn times) to be considered separately because: (i) in practical situations, one model could be more adapted than some other; (ii) different models will yield specific parameter estimators, so it is important to study them separately.

The paper is organized as follows. The next section Semi-Markov models describes the semiMarkov models used in this package. Section The SMM package illustrates the different functions of the SMM package. We end the paper by presenting some concluding remarks on this $\mathrm{R}$ package.

\section{Semi-Markov models}

Let us consider a random system with finite state space $E=\{1, \ldots, s\}, s<\infty$. Let $(\Omega, \mathcal{A}, \mathbb{P})$ be a probability space and assume that the time evolution of the system is governed by a stochastic process $Y=\left(Y_{k}\right)_{k \in \mathbb{N}^{*}}$, defined on $(\Omega, \mathcal{A}, \mathbb{P})$ with values in $E$; that is to say that $Y_{k}$ gives the state of the system at time $k$. Let $T=\left(T_{m}\right)_{m \in \mathbb{N}^{*}}$, defined on $(\Omega, \mathcal{A}, \mathbb{P})$ with values in $\mathbb{N}$, be the successive time points when state changes in $\left(Y_{k}\right)_{k \in \mathbb{N}^{*}}$ occur (the jump times) and let also $J=\left(J_{m}\right)_{m \in \mathbb{N}^{*}}$, defined on $(\Omega, \mathcal{A}, \mathbb{P})$ with values in $E$, be the successively visited states at these time points. The relation between the process $Y$ and the process $J$ of the successively visited states is given by $Y_{k}=J_{N(k)}$, or, equivalently, $J_{m}=Y_{T_{m}}, m, k \in \mathbb{N}$, where $N(k):=\max \left\{m \in \mathbb{N} \mid T_{m} \leq k\right\}$ is the discrete-time counting process of the number of jumps in $[1, k] \subset \mathbb{N}$.

In this paper we consider four different semi-Markov models corresponding to the following four types of sojourn times.

- Sojourn times depending on the current state and on the next state:

$$
f_{i j}(k)=\mathbb{P}\left(T_{m+1}-T_{m}=k \mid J_{m}=i, J_{m+1}=j\right) ;
$$

- Sojourn times depending only on the current state:

$$
f_{i \bullet}(k)=\mathbb{P}\left(T_{m+1}-T_{m}=k \mid J_{m}=i\right) ;
$$


- Sojourn times depending only on the next state to be visited:

$$
f_{\bullet j}(k)=\mathbb{P}\left(T_{m+1}-T_{m}=k \mid J_{m+1}=j\right) ;
$$

- Sojourn times depending neither on the current state nor on the next state:

$$
f(k)=\mathbb{P}\left(T_{m+1}-T_{m}=k\right) .
$$

Note that the sojourn times of the type $f_{i \bullet}(\cdot), f_{\bullet j}(\cdot)$ or $f(\cdot)$ are particular cases of the general type $f_{i j}(\cdot)$. Nonetheless, in some specific applications, particular cases can be important because they are adapted to the phenomenon under study; that is the reason why we investigate these cases separately.

\section{General case: sojourn times of the type $f_{i j}($.}

Definition 1 (semi-Markov chain SMC and Markov renewal chain MRC). If we have

$$
\mathbb{P}\left(J_{m+1}=j, T_{m+1}-T_{m}=k \mid J_{m}=i, J_{m-1}, \ldots, J_{1}, T_{m}, \ldots, T_{1}\right)=\mathbb{P}\left(J_{m+1}=j, T_{m+1}-T_{m}=k \mid J_{m}=i\right),
$$

then $Y=\left(Y_{k}\right)_{k}$ is called a semi-Markov chain $(S M C)$ and $(J, T)=\left(J_{m}, T_{m}\right)_{m}$ is called a Markov renewal chain $(M R C)$.

All along this paper we assume that the MRC or SMC are homogeneous with respect to the time in the sense that Equation (1) is independent of $m$.

Note that if $(J, T)$ is a MRC, then it can be proved that $J=\left(J_{m}\right)_{m \in \mathbb{N}^{*}}$ is a Markov chain with state space $E$, called the embedded Markov chain of the MRC ( J,T) (or of the SMC Y).

Definition 2. For a semi-Markov chain we define:

- the semi-Markov kernel $\left(q_{i j}(k)\right)_{i, j \in E, k \in \mathbb{N}}$,

$$
q_{i j}(k)=\mathbb{P}\left(J_{m+1}=j, T_{m+1}-T_{m}=k \mid J_{m}=i\right) ;
$$

- the initial distribution $\left(\mu_{i}\right)_{i \in E}$,

$$
\mu_{i}=\mathbb{P}\left(J_{1}=i\right)=\mathbb{P}\left(Y_{1}=i\right) ;
$$

- the transition matrix $\left(p_{i j}\right)_{i, j \in E}$ of the embedded Markov chain $J=\left(J_{m}\right)_{m}$,

$$
p_{i j}=\mathbb{P}\left(J_{m+1}=j \mid J_{m}=i\right) ;
$$

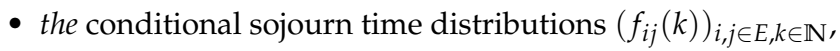

$$
f_{i j}(k)=\mathbb{P}\left(T_{m+1}-T_{m}=k \mid J_{m}=i, J_{m+1}=j\right) .
$$

Note that

$$
q_{i j}(k)=p_{i j} f_{i j}(k) .
$$

Clearly, a semi-Markov chain is uniquely determined a.s. by an initial distribution $\left(\mu_{i}\right)_{i \in E}$ and a semi-Markov kernel $\left(q_{i j}(k)\right)_{i, j \in E, k \in \mathbb{N}}$ or, equivalently, by an initial distribution $\left(\mu_{i}\right)_{i \in E}$, a Markov transition matrix $\left(p_{i j}\right)_{i, j \in E}$ and conditional sojourn time distributions $\left(f_{i j}(k)\right)_{i, j \in E, k \in \mathbb{N}}$.

Other assumptions we make are: (i) We do not allow transitions to the same state, i.e., $p_{i i}=0$ for all $i \in E$, or equivalently $q_{i i}(k)=0$, for all $i \in E, k \in \mathbb{N}$; (ii) We assume that there are not instantaneous transitions, that is $q_{i j}(0) \equiv 0$ or equivalently $f_{i j}(0) \equiv 0$ for all $i, j \in E$; note that this implies that $T$ is a strictly increasing sequence.

For the conditional sojourn time distributions, one can consider the associated cumulative distribution function defined by

$$
F_{i j}(k):=\mathbb{P}\left(T_{m+1}-T_{m} \leq k \mid J_{m}=i, J_{m+1}=j\right)=\sum_{t=1}^{k} f_{i j}(t) .
$$

For any distribution function $F(\cdot)$ we can consider the associated survival/reliability function defined by

$$
\bar{F}(k):=1-F(t) .
$$


Consequently we have

$$
\bar{F}_{i j}(k):=\mathbb{P}\left(T_{m+1}-T_{m}>k \mid J_{m}=i, J_{m+1}=j\right)=1-\sum_{t=1}^{k} f_{i j}(t)=\sum_{t=k+1}^{\infty} f_{i j}(t) .
$$

Particular cases: sojourn times of the type $f_{i .}(),. f_{. j}($.$) and f($.

We have considered up to here general semi-Markov models with the semi-Markov kernel of the type given in (2). Particular types of this semi-Markov model can be taken into account, by considering particular cases of holding time distributions $f_{i j}(k)$, where these distributions depend only on the current state $i$, or only on the next state $j$, or neither on $i$ nor on $j$. For each case, let us define the semi-Markov kernel and the distribution function associated to the sojourn time distribution.

- Sojourn times depending only on the current state:

$$
\begin{aligned}
& q_{i j}(k):=p_{i j} f_{i \bullet}(k), \text { where } \\
& f_{i \bullet}(k)=\mathbb{P}\left(T_{m+1}-T_{m}=k \mid J_{m}=i\right)=\sum_{v \in E} p_{i v} f_{i v}(k), \\
& F_{i \bullet}(k):=\mathbb{P}\left(T_{m+1}-T_{m} \leq k \mid J_{m}=i\right)=\sum_{t=1}^{k} f_{i \bullet}(t)=\sum_{t=1}^{k} \sum_{v \in E} p_{i v} f_{i v}(t) .
\end{aligned}
$$

- Sojourn times depending only on the next state:

$$
\begin{aligned}
q_{i j}(k) & :=p_{i j} f_{\bullet j}(k), \text { where } \\
f_{\bullet j}(k) & =\mathbb{P}\left(T_{m+1}-T_{m}=k \mid J_{m+1}=j\right), \\
F_{\bullet j}(k) & :=\mathbb{P}\left(T_{m+1}-T_{m} \leq k \mid J_{m+1}=j\right)=\sum_{t=1}^{k} f_{\bullet j}(t) .
\end{aligned}
$$

- Sojourn times depending neither on the current state nor on the next state:

$$
\begin{aligned}
q_{i j}(k) & :=p_{i j} f(k), \text { where } \\
f(k) & =\mathbb{P}\left(T_{m+1}-T_{m}=k\right) . \\
F(k) & :=\mathbb{P}\left(T_{m+1}-T_{m} \leq k\right)=\sum_{t=1}^{k} f(t) .
\end{aligned}
$$

We also denote the associated survival/reliability functions respectively by $\bar{F}_{i \bullet}(k), \bar{F}_{\bullet j}(k), \bar{F}(k)$.

\section{The SMM package}

The SMM R package is mainly devoted to the simulation and estimation of discrete-time semi-Markov models in different cases by the two following functions:

$\operatorname{simulSM()}$ for the simulation of sequences from a semi-Markov model:

- One or several trajectories

- According to classical distributions for the sojourn times (Uniform, Geometric, Poisson, Discrete Weibull and Negative Binomial) or according to distributions given by the user

- Four different types of censoring mechanisms: censoring at the beginning of sample paths, censoring at the end, censoring at the beginning and at the end, no censoring

- Four different types of sojourn times: depending on the current state and on the next state, depending only on the current state, depending only on the next state, depending neither on the current state nor on the next state

estimSM() for the estimation of model parameters:

- One or several trajectories

- Parametric (Uniform, Geometric, Poisson, Discrete Weibull and Negative Binomial) or non-parametric distributions for the sojourn times

- Four different types of censoring mechanisms: censoring at the beginning of sample paths, censoring at the end, censoring at the beginning and at the end, no censoring 
- Four different types of sojourn times: depending on the current state and on the next state, depending only on the current state, depending only on the next state, depending neither on the current state nor on the next state

As the Negative Binomial distribution and the Discrete Weibull distribution can have several different parameterizations, note that we have considered the following ones in our package:

- Discrete Weibull of type I with the density $f(x)=q^{(x-1)^{\beta}}-q^{x^{\beta}}$ for $x=1,2, \ldots, n$ with $n>0, q$ is the first parameter, $0<q<1$, and $\beta$ is the second parameter, $\beta>0$;

- Negative Binomial with the density $f(x)=\frac{\Gamma(x+\alpha)}{\Gamma(\alpha) x !}\left(\frac{\alpha}{\alpha+\mu}\right)^{\alpha}\left(\frac{\mu}{\alpha+\mu}\right)^{x}$ for $x=$ $0,1, \ldots, n$ with $n>0, \Gamma$ is the Gamma function, $\alpha$ is the parameter of overdispersion, $\alpha>0$, and $\mu$ is the mean, $\mu \in \mathbb{R}$.

In order to avoid any confusion, note also that the expressions of the different densities considered in this package are also provided in the corresponding manual.

The SMM R package is also devoted to the simulation and estimation of discrete-time Markov models by the two following functions:

simulMk() for the simulation of sequences from a $k$ th order Markov model;

estimMk() for the estimation of the parameters of the model.

All the different possibilities of the package are illustrated in Figure 1.

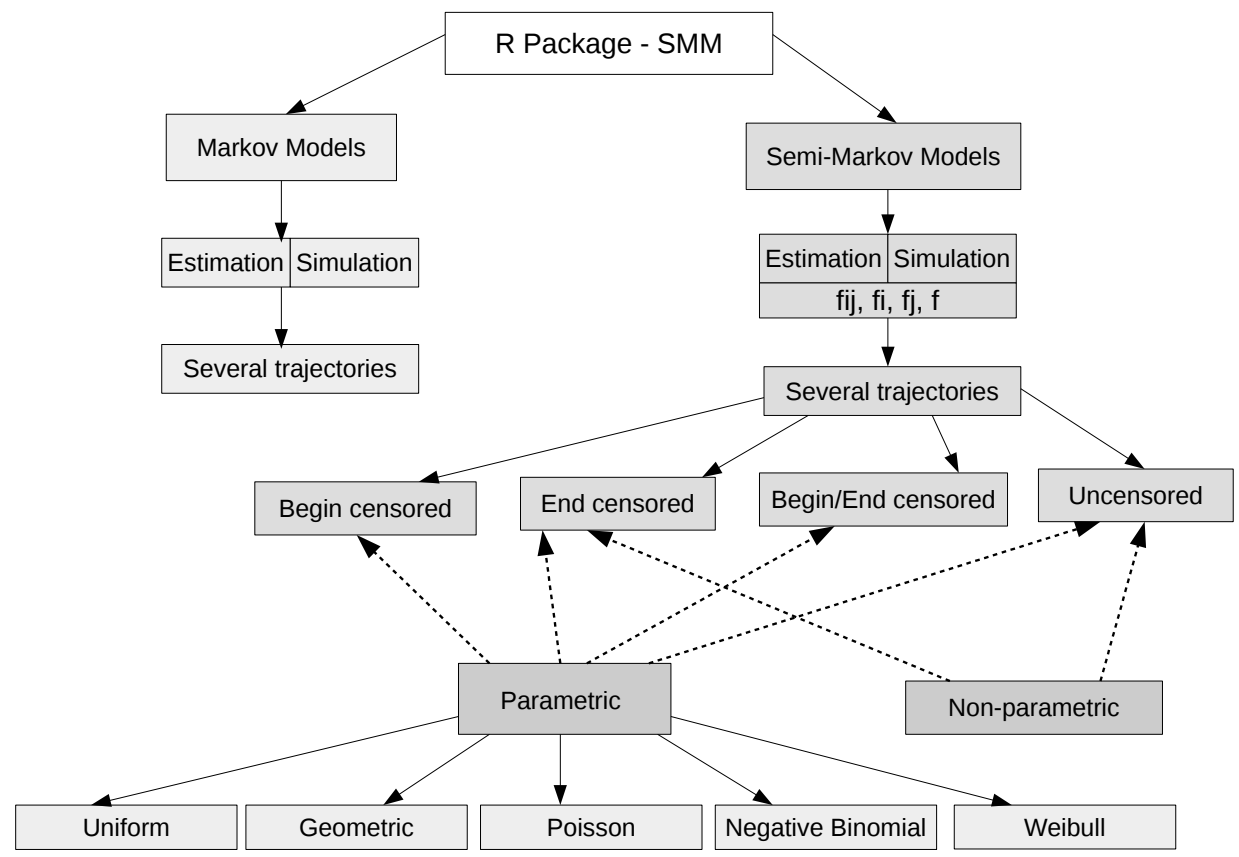

Figure 1: Schema of the SMM package.

\section{Simulation of semi-Markov models}

\section{Parametric simulation: according to classical distributions}

In this part, we will consider the simulation according to classical distributions.

Parameters: This simulation is carried out by the function simulSM(). The different parameters of the function are:

- E: Vector of state space of length $S$

- NbSeq: Number of simulated sequences 
- lengthSeq: Vector containing the lengths of each simulated sequence

- TypeSojournTime: Type of sojourn time; it can be "fij", "fi", "fj" or "f" according to the four cases previously discussed

- init: Vector of initial distribution of length $S$

- Ptrans: Matrix of transition probabilities of the embedded Markov chain $J=\left(J_{m}\right)_{m}$ of size $S \times S$

- distr: Sojourn time distributions:

- is a matrix of distributions of size $S \times S$ if TypeSojournTime is equal to "fij",

- is a vector of distributions of size $S$ if TypeSojournTime is equal to " $f i$ " or " $f j$ ",

- is a distribution if TypeSojournTime is equal to " $f$ ",

where the distributions to be used can be one of "uniform", "geom", "pois", "weibull" or "nbinom".

- param: Parameters of sojourn time distributions:

- is an array of parameters of size $S \times S \times 2$ if TypeSojournTime is equal to " $\mathrm{fij}$ "

- is a matrix of parameters of size $S \times 2$ if TypeSojournTime is equal to " $f \mathrm{i}$ " or " $\mathrm{fj}$ "

- is a vector of parameters if TypeSojournTime is equal to " $f$ "

- cens. beg: Type of censoring at the beginning of sample paths; 1 (if the first sojourn time is censored) or 0 (if not). All the sequences must be censored in the same way.

- cens. end: Type of censoring at the end of sample paths; 1 (if the last sojourn time is censored) or 0 (if not). All the sequences must be censored in the same way.

- File. out: Name of fasta file for saving the sequences; if File. out = NULL, no file is created. A fasta file is a simple text file containing sequences only described by one description line beginning by a ">": an example is given in Figure 2.

$>$ sequence 1

aaacgtacgagtcgatcgatcgactcgatcgtacgtacggt

$>$ sequence 2

acgtattacgatgctagctaggttggggactgcatgcatgaatgagcgatc

Figure 2: Example of a fasta file.

The R commands below generate three sequences of size 1000,10000 and 2000 respectively with the finite state space $E=\{a, c, g, t\}$, where the sojourn times depend on the current state and on the next state.

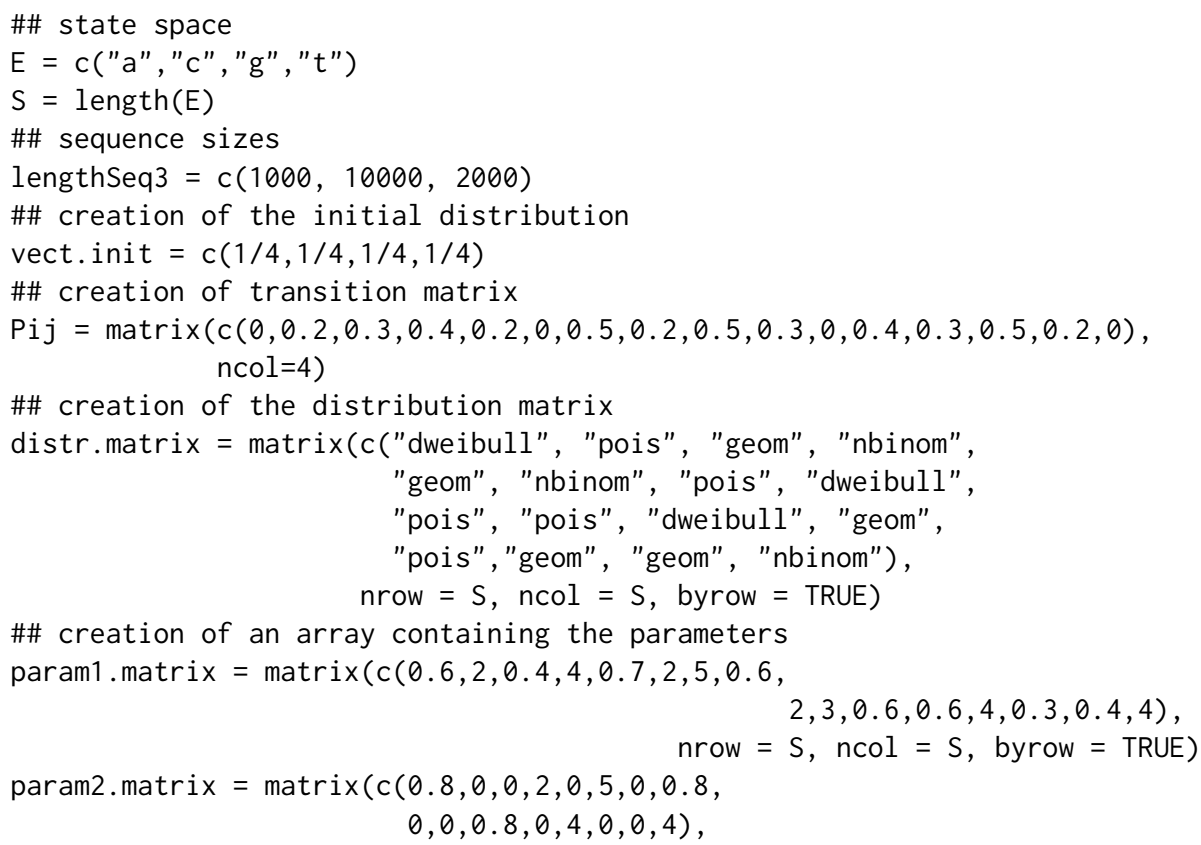


param. array $=\operatorname{array}(\mathrm{c}($ param1.matrix, param2.matrix), $\mathrm{c}(\mathrm{S}, \mathrm{S}, 2))$

\#\# for the reproducibility of the results

set. seed(1)

\#\# simulation of 3 sequences

seq3 $=\operatorname{simulSM}(E=E, N b S e q=3$, lengthSeq $=$ lengthSeq 3, TypeSojournTime $=$ "fij", init $=$ vect.init,

Ptrans $=$ Pij, distr $=$ distr. matrix, param $=$ param. array

File. out $=$ "seq3.txt")

First, note that in this simulation, the parameters cens. beg and cens. end are equal to 0 , that is to say the simulated sequences are not censored.

Second, note also that the parameters of the distributions are given in the following way: for example, $f_{13}(\cdot)$ is Geometric distribution with parameter 0.4 , while $f_{14}(\cdot)$ is Negative Binomial with parameters 4 and 2 . In other words, the parameters of $f_{13}(\cdot)$ are given in the vector param. array $[1,3$, that is equal to $(0.4,0)$ and the parameters of $f_{14}(\cdot)$ are given in the vector param. array $[1,4$,$] that$ is equal to $(4,2)$; that means that if a distribution has only 1 parameter, the corresponding vector of parameters will have 0 on the second position.

Values: The function simulSM() returns a list of simulated sequences. These sequences can be saved in a fasta file by using the parameter File. out.

seq3[[1]][1:15]

[1] "g" "g" "g" "g" "c" "c" "c" "a" "a" "a" "c" "c" "c" "g" "g"

\section{Non-parametric simulation: according to distributions given by the user}

Now we will consider the simulation according to distributions given by the user.

Parameters: This simulation is carried out by the function simulSM(). The different parameters of the function are:

- E: Vector of state space of length $S$

- NbSeq: Number of simulated sequences

- lengthSeq: Vector containing the lengths of each simulated sequence

- TypeSojournTime: Type of sojourn time; it can be "fij", "fi", "fj" or "f" according to the four cases previously discussed

- init: Vector of initial distribution of length $S$

- Ptrans: Matrix of transition probabilities of the embedded Markov chain $J=\left(J_{m}\right)_{m}$ of size $S \times S$

- laws: Sojourn time distributions introduced by the user:

- is an array of size $S \times S \times K \max$ if TypeSojournTime is equal to " $\mathrm{fij}$ ",

- is a matrix of size $S \times K \max$ if TypeSojournTime is equal to " $\mathrm{fi}$ " or " $\mathrm{fj}$ ",

- is a vector of length Kmax if TypeSojournTime is equal to " $f$ ",

where Kmax is the maximum length for the sojourn times.

- cens. beg: Type of censoring at the beginning of sample paths; 1 (if the first sojourn time is censored) or 0 (if not). All the sequences must be censored in the same way.

- cens. end: Type of censoring at the end of sample paths; 1 (if the last sojourn time is censored) or 0 (if not). All the sequences must be censored in the same way.

- File. out: Name of fasta file for saving the sequences; if File. out = NULL, no file is created.

The R commands below generate three sequences of size 1000,10000 and 2000 respectively with the finite state space $E=\{a, c, g, t\}$, where the sojourn times depend only on the next state.

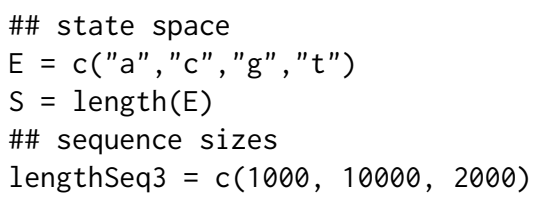




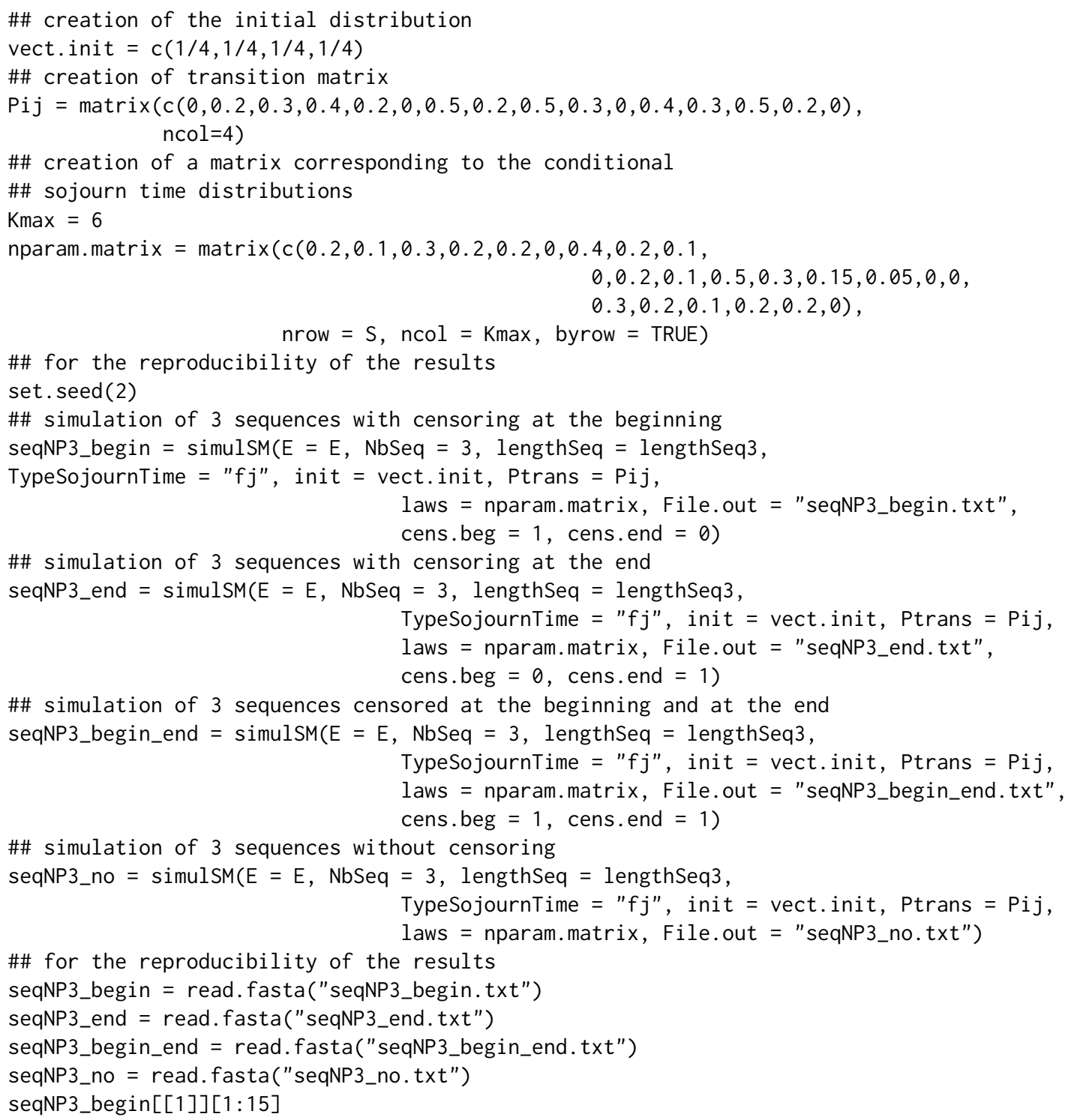

Note that in this simulation all the different censoring mechanisms are considered.

Values: The function simulSM() returns a list of simulated sequences. These sequences can be saved in a fasta file by using the parameter File. out.

seqNP3_begin[[1]][1:15]

[1] "c" "g" "g" "g" "g" "g" "g" "c" "c" "c" "a" "a" "a" "a" "a"

\section{Estimation of semi-Markov models}

In this subsection we explain and illustrate the estimation of a semi-Markov model in the parametric and non-parametric cases.

\section{Parametric estimation of semi-Markov models}

We will consider the distributions $f_{i j}(k)=f_{i j}\left(k ; \theta_{i j}\right)$ depending on unknown parameters $\theta_{i j} \in \mathbb{R}^{m_{i j}}$, where the dimension of parameters set $m_{i j}$ is known; no assumptions are made on $\left(p_{i j}\right)_{i j}$. From the data, we want to estimate $p_{i j}$ and $\theta_{i j}, i, j \in E$. Let us assume that we have at our disposal several independent sample paths of a semi-Markov chain, say $L$, each of them of length $n_{l}, l=1, \ldots, L$, censored at the beginning and at the end of the trajectory, i.e.,

$$
y_{1}^{l}, y_{2}^{l}, \ldots, y_{n_{1}}^{l}
$$


or, equivalently,

$$
j_{0}^{l}, k_{0}^{l}, j_{1}^{l}, k_{1}^{l}, j_{2}^{l}, k_{2}^{l}, \ldots, j_{t^{l}}^{l}, k_{t^{l}}^{l}, j_{t^{l}+1}^{l}, k_{t^{l}+1}^{l}
$$

with $\sum_{i=0}^{t^{l}+1} k_{i}^{l}=n_{l}$, where $j_{0}^{l}, \ldots, j_{t^{l}+1}^{l}$ are the successive distinct visited states, $k_{0}^{l}$ is the first sojourn time, assumed to be right censored, $k_{t^{l}+1}^{l}$ is the last sojourn time, assumed also to be right censored, while $k_{1}^{l}, \ldots, k_{t^{l}}^{l}$ are the other successive sojourn times, assumed to be complete (observed, non censored).

To estimate the parameters of model, we use the maximum likelihood estimation:

$$
\begin{aligned}
& \underset{p_{u v}, \theta_{u v} ; u, v \in E}{\operatorname{argmax}}\left(l\left(p_{u v}, \theta_{u v} ; u, v \in E\right)\right) \\
& =\left(\underset { p _ { u v } , \theta _ { u v } ; v \in E } { \operatorname { a r g m a x } } \left(\sum_{v \in E} N_{u v}\left(L, n_{1: L}\right) \log \left(p_{u v}\right)+\sum_{v \in E} \sum_{k=1}^{\max _{l}\left(n_{l}\right)} N_{u v}\left(k ; L, n_{1: L}\right) \log \left(f_{u v}\left(k ; \theta_{u v}\right)\right)\right.\right. \\
& +\sum_{v \in E} \sum_{k=1}^{\max _{l}\left(n_{l}\right)} \bar{N}_{u v}^{b}(k ; L) \log \left(\bar{F}_{u v}\left(k ; \theta_{u v}\right)\right) \\
& \left.\left.+\sum_{k=1}^{\max _{l}\left(n_{l}\right)} \bar{N}_{u \bullet}^{e}(k ; L) \log \left(1-\sum_{m=1}^{k} \sum_{v \in E} p_{u v} f_{u v}\left(m ; \theta_{u v}\right)\right)\right)_{u \in E}\right)
\end{aligned}
$$

where we introduced the following counting processes

$$
\begin{aligned}
N_{i j}\left(L, n_{1: L}\right) & =\sum_{l=1}^{L} \sum_{m=1}^{N^{l}\left(n_{l}\right)-1} \mathbb{1}_{\left\{J_{m}^{l}=i ; J_{m+1}^{l}=j\right\}^{\prime}} \\
N_{i j}\left(k ; L, n_{1: L}\right) & =\sum_{m=1}^{N^{l}\left(n_{l}\right)-1} \mathbb{1}_{\left\{J_{m}^{l}=i ; J_{m+1}^{l}=j ; T_{m+1}^{l}-T_{m}^{l}=k\right\}^{\prime}}, \\
\bar{N}_{i j}^{b}(k ; L) & =\sum_{l=1}^{L} \mathbb{1}_{\left\{J_{0}^{l}=i ; J_{1}^{l}=j ; T_{1}^{l}-T_{0}^{l}>k\right\}^{\prime}} \\
\bar{N}_{i \bullet}^{e}(k ; L) & =\sum_{l=1}^{L} \mathbb{1}_{\left\{J_{N^{l}\left(n_{l}\right)}^{l}=i, X_{N^{l}\left(n_{l}\right)+1}^{l}>k\right\}^{\prime}}>
\end{aligned}
$$

where

$$
N^{l}\left(n_{l}\right)=\max \left\{m \in \mathbb{N} \mid T_{m}^{l} \leq n_{l}\right\}
$$

is the counting process of jump number in $\left[1 ; n_{l}\right]$ of the trajectory $l$.

Note that:

- $N_{i j}\left(L, n_{1: L}\right)$ represents the number of transitions from state $i$ to state $j$ along the $L$ sample paths;

- $N_{i j}\left(k ; L, n_{1: L}\right)$ represents the number of transitions from state $i$ to state $j$ along the $L$ sample paths, with sojourn time of length $k$ in state $i$;

- $\bar{N}_{i j}^{b}(k ; L)$ represents the number of trajectories starting in state $i$, with a next transition to state $j$ and censored sojourn time in state $i$ greater than $k$;

- $\bar{N}_{i \bullet}^{e}(k ; L)$ represents the number of trajectories ending in state $i$ with a censored sojourn time in state $i$ greater than $k$.

Note also that in the expression (6) of the log-likelihood, the first two terms correspond to the transition probabilities and the observed (non censored) sojourn times, the third term is the contribution to the likelihood of the first sojourn times, assumed to be right censored, while the last term is the contribution to the likelihood of the last sojourn times, assumed to be right censored.

Up to here we presented the estimation for the general case, taking into account the censoring at the beginning and at the end and the sojourn times depending on the current state and on the next state. Thus the maximization problem (6) is written with the sojourn times depending on the current state and on the next state (the general model of the type $q_{i j}(k)=p_{i j} f_{i j}(k)$ given in (2)), but the 
different cases of sojourn times are written and coded in the package. Note also that different types of censoring are also written and coded in the package.

Parameters: The estimation is carried out by the function estimSM(). The different parameters of the function are:

- file: Path of the fasta file which contains the sequences from which to estimate

- seq: List of the sequence(s) from which to estimate

- E: Vector of state space of length $S$

- TypeSojournTime: Type of sojourn time; it can be "fij", "fi", "fj" or "f" according to the four cases previously discussed

- distr: Sojourn time distributions:

- is a matrix of distributions of size $S \times S$ if TypeSojournTime is equal to " $f i j "$,

- is a vector of distributions of size $S$ if TypeSojournTime is equal to " $f i$ " or " $f j$ ",

- is a distribution if TypeSojournTime is equal to " $f$ ",

where the distributions to be used can be one of "uniform", "geom", "pois", "weibull" or "nbinom".

- cens. beg: Type of censoring at the beginning of sample paths; 1 (if the first sojourn time is censored) or 0 (if not). All the sequences must be censored in the same way.

- cens. end: Type of censoring at the end of sample paths; 1 (if the last sojourn time is censored) or 0 (if not). All the sequences must be censored in the same way.

Note that the sequences from which we estimate can be given either as an R list (seq argument) or as a file in fasta format (file argument).

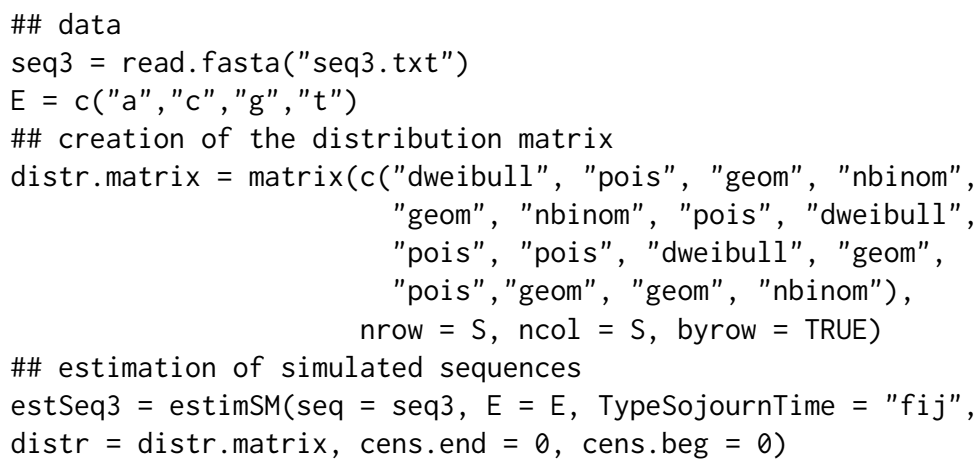

Here, we estimate simulated sequences with no censoring. The estimation performed will correspond to the likelihood given in (6), without the third and forth terms.

Values: The function estimSM() returns a list containing:

- init: Vector of size $S$ with estimated initial probabilities of the semi-Markov chain estSeq3init

[1] $0.0000000 \quad 0.0000000 \quad 0.33333330 .6666667$

- Ptrans: Matrix of size $S \times S$ with estimated transition probabilities of the embedded Markov chain $J=\left(J_{m}\right)_{m}$

estSeq3 \$Ptrans

$[, 1] \quad[, 2] \quad[, 3] \quad[, 4]$

$[1] \quad 0.0000000 \quad 0.2263948 \quad$,

$[2] \quad 0.21344720 .0000000 \quad$,

$[3] \quad 0.2900627 \quad 0.47090420 .0000000 \quad$,

$\left[\begin{array}{lllllll}4,] & 0.4108761 & 0.2024169 & 0.3867069 & 0.0000000\end{array}\right.$

- param: Array with estimated parameters of the sojourn time distributions 
estSeq3 \$param

, 1

$$
[, 1] \quad[, 2] \quad[, 3] \quad[, 4]
$$

$[1] \quad 0.00000001 .78672990 .411398 \quad$,

$[2] \quad 0.6920415 \quad 0.0000000 \quad 4.745387 \quad$,

$[3] \quad 2.0123457 \quad 3.0722433 \quad 0.000000 \quad$,

$\left[\begin{array}{lllllll} & 3.8088235 & 0.3068702 & 0.392237 & 0.0000000\end{array}\right.$

$, \quad, 2$

$\begin{array}{rrrrr} & {[, 1]} & {[, 2]} & {[, 3]} & {[, 4]} \\ {[1,]} & 0 & 0 & 0 & 2.1043710 \\ {[2,]} & 0 & 0 & 0 & 0.8425902 \\ {[3,]} & 0 & 0 & 0 & 0.0000000 \\ {[4,]} & 0 & 0 & 0 & 0.0000000\end{array}$

Note that, for example, estSeq3 $\$$ param $[1,3$,$] is the vector containing the parameters of the$ distribution $f_{13}(\cdot)$.

- q: Array of size $S \times S \times K \max$ with estimated semi-Markov kernel

estSeq3 $\$ \mathrm{q}[,, 1: 3]$

, , 1

$$
[, 1] \quad[, 2] \quad[, 3] \quad[, 4]
$$

$[1] \quad 0.00000000 \quad 0.03792273 \quad$,

$[2] \quad 0.14771431 \quad 0.00000000 \quad$,

$\left[\begin{array}{lllllll}{[3,]} & 0.03877405 & 0.02181093 & 0.00000000 & 0.13695675\end{array}\right.$

$\left[\begin{array}{llllllll}4,] & 0.00911087 & 0.06211573 & 0.15168076 & 0.00000000\end{array}\right.$

$, \quad, 2$

$$
[, 1] \quad[, 2] \quad[, 3] \quad[, 4]
$$

$[1] \quad 0.00000000 \quad 0.06775768 \quad 0.12003558 \quad$,

$[2] \quad 0.04548987 \quad 0.00000000 \quad$,

$[3] \quad 0.07802680 \quad 0.067008490 .00000000 \quad$,

$\left[\begin{array}{llllll}4,] & 0.03470170 & 0.04305426 & 0.09218596 & 0.00000000\end{array}\right.$

,$\quad 3$

$[, 1] \quad[, 2] \quad[, 3] \quad[, 4]$

$[1] \quad 0.00000000 \quad 0.060532330 .07065318 \quad$,

$[2] \quad 0.01400899 \quad 0.00000000 \quad$,

$[3] \quad 0.07850845 \quad 0.10293320 \quad 0.00000000 \quad$,

$\left[\begin{array}{llllll}4, & 0.06608632 & 0.02984219 & 0.05602722 & 0.00000000\end{array}\right.$

Note that, for example, $q_{13}(2)=\mathbb{P}\left(J_{m+1}=3, T_{m+1}-T_{m}=2 \mid J_{m}=1\right)=0.12192187$.

\section{Non-parametric estimation of semi-Markov models}

Here we will consider two types of estimation for semi-Markov chains: a direct estimation, obtaining thus empirical estimators (in fact, approached MLEs), cf. Barbu and Limnios (2006), Barbu and Limnios (2008) and an estimation based on a couple Markov chain associated to the semi-Markov chain (see Trevezas and Limnios, 2011).

\section{No censoring: direct estimation}

Let $\left\{Y_{1}, Y_{2}, \ldots, Y_{n}\right\}$ be a trajectory of a semi-Markov chain $Y=\left(Y_{n}\right)_{n \in \mathbb{N}}$, censored at an arbitrary fixed time $n$.

- The case $q_{i j}(k)=p_{i j} f_{i j}(k)$ : The maximum likelihood estimators are

$$
\widehat{p_{i j}}(n)=\frac{N_{i j}(n)}{N_{i \bullet}(n)}, \quad \widehat{f_{i j}}(k ; n)=\frac{N_{i j}(k ; n)}{N_{i j}(n)}, \quad \widehat{q_{i j}}(k ; n)=\frac{N_{i j}(k ; n)}{N_{i \bullet}(n)} .
$$


where $N_{i j}(n)=\sum_{m=1}^{N(n)-1} \mathbb{1}_{\left\{J_{m}=i ; J_{m+1}=j\right\}}, N_{i \bullet}(n)=\sum_{m=1}^{N(n)-1} \mathbb{1}_{\left\{J_{m}=i\right\}}$, $N_{i j}(k ; n)=\sum_{m=1}^{N(n)-1} \mathbb{1}_{\left\{J_{m}=i ; J_{m+1}=j ; T_{m+1}-T_{m}=k\right\}}$.

- The case $q_{i j}(k)=p_{i j} f_{i \bullet}(k)$ : The maximum likelihood estimators are

$$
\widehat{p_{i j}}(n)=\frac{N_{i j}(n)}{N_{i \bullet}(n)}, \quad \widehat{f_{i \bullet}}(k ; n)=\frac{N_{i \bullet}(k ; n)}{N_{i j}(n)}, \quad \widehat{q_{i j}}(k ; n)=\frac{N_{i \bullet}(k ; n)}{N_{i \bullet}(n)},
$$

where $N_{i \bullet}(k ; n)=\sum_{m=1}^{N(n)-1} \mathbb{1}_{\left\{J_{m}=i ; T_{m+1}-T_{m}=k\right\}}$.

- The case $q_{i j}(k)=p_{i j} f_{\bullet j}(k)$ : The maximum likelihood estimators are

$$
\widehat{p_{i j}}(n)=\frac{N_{i j}(n)}{N_{i \bullet}(n)}, \quad \widehat{f_{\bullet j}}(k ; n)=\frac{N_{\bullet j}(k ; n)}{N_{i j}(n)}, \quad \widehat{q_{i j}}(k ; n)=\frac{N_{\bullet j}(k ; n)}{N_{i \bullet}(n)},
$$

where $N_{\bullet j}(k ; n)=\sum_{m=1}^{N(n)-1} \mathbb{1}_{\left\{J_{m+1}=j ; T_{m+1}-T_{m}=k\right\}}$.

- The case $q_{i j}(k)=p_{i j} f(k)$ : The maximum likelihood estimators are

$$
\widehat{p_{i j}}(n)=\frac{N_{i j}(n)}{N_{i \bullet}(n)}, \quad \widehat{f}(k ; n)=\frac{N(k ; n)}{N_{i j}(n)}, \quad \widehat{q_{i j}}(k ; n)=\frac{N(k ; n)}{N_{i \bullet}(n)},
$$

where $N(k ; n)=\sum_{m=1}^{N(n)-1} \mathbb{1}_{\left\{T_{m+1}-T_{m}=k\right\}}$.

Censoring: couple Markov chain For a semi-Markov chain $Y=\left(Y_{n}\right)_{n \in \mathbb{N}}$, let $U=\left(U_{n}\right)_{n \in \mathbb{N}}$ be the backward recurrence time of the SMC, defined by

$$
U_{n}:=n-T_{N(n)} .
$$

We can show (cf. Limnios and Oprisan, 2001) that the chain $(Y, U)=\left(Y_{n}, U_{n}\right)_{n \in \mathbb{N}}$ is a Markov chain with state space $E \times \mathbb{N}$. We will denote its transition matrix by $\widetilde{\mathbf{p}}:=\left(p_{\left(i, t_{1}\right)\left(j, t_{2}\right)}\right)_{i, j \in E, t_{1}, t_{2} \in \mathbb{N}}$.

The maximum likelihood estimators of $q_{i j}(k)$ (Trevezas and Limnios, 2011) are given by

$$
\widehat{q_{i j}}(k ; n)=\widehat{p}_{(i, k-1)(j, 0)}(n) \prod_{t=0}^{k-2} \widehat{p}_{(i, t)(i, t+1)}(n),
$$

where $\widehat{p}_{\left(i, t_{1}\right)\left(j, t_{2}\right)}(n)$ represents the classical MLE of the transition probability $p_{\left(i, t_{1}\right)\left(j, t_{2}\right)}$. Thus we obtain the corresponding estimator of $p_{i j}$

$$
\widehat{p}_{i j}=\sum_{k=0}^{\infty} \widehat{q}_{i j}(k)
$$

In order to compute the estimators of the sojourn times, we consider the four different types of semi-Markov kernels defined in Equations (2), (3), (4) and (5).

Parameters: The estimation is carried out by the function estimSM() and several parameters must be given.

- file: Path of the fasta file which contains the sequences from which to estimate

- seq: List of the sequence(s) from which to estimate

- E: Vector of state space of length $S$

- TypeSojournTime: Type of sojourn time; always equal to "NP" for the non-parametric estimation

- cens beg: Type of censoring at the beginning of sample paths; 1 (if the first sojourn time is censored) or 0 (if not). All the sequences must be censored in the same way.

- cens. end: Type of censoring at the end of sample paths; 1 (if the last sojourn time is censored) or 0 (if not). All the sequences must be censored in the same way.

Note that the sequences from which we estimate can be given either as an R list (seq argument) or as a file in fasta format (file argument). The parameter distr is always equal to "NP".

\#\# data

seqNP3_no = read.fasta("seqNP3_no.txt") 
$E=c(" a ", " c ", " g ", " t ")$

\#\# estimation of simulated sequences

estSeqNP3= estimSM(seq = seqNP3_no, E = E, TypeSojournTime = "fj", distr $=$ "NP", cens.end $=0$, cens. beg $=0$ )

Here, we estimate simulated sequences with no censoring.

Values: The function estimSM() returns a list containing:

- init: Vector of size $S$ with estimated initial probabilities of the semi-Markov chain estSegNP3\$init [1] $0.0000000 \quad 0.6666667 \quad 0.33333330 .0000000$

- Ptrans: Matrix of size $S \times S$ with estimated transition probabilities of the embedded Markov chain $J=\left(J_{m}\right)_{m}$ estSeqNP3\$Ptrans
$[, 1]$
$[, 2]$
$[, 3]$
[, 4]

$\left[\begin{array}{lllllll}{[1,]} & 0.0000000 & 0.2051948 & 0.5090909 & 0.2857143\end{array}\right.$

$[2] \quad 0.19381790 .0000000 \quad$,

$[3] \quad 0.30101690 .4874576 \quad 0.0000000 \quad$,

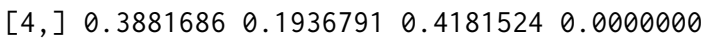

- laws: Array of size $S \times S \times K \max$ with estimated values of the sojourn time distributions estSeqNP3\$laws[, , $1: 2]$ , 1
$[, 1]$
$[, 2]$
$[, 3]$
$[, 4]$

$\left[\begin{array}{lllllll}{[1,]} & 0.0000000 & 0.3941423 & 0.4728997 & 0.2939271\end{array}\right.$

$\left[\begin{array}{lllllll}{[2,]} & 0.1896104 & 0.0000000 & 0.4728997 & 0.2939271\end{array}\right.$

$\left[\begin{array}{lllllll}{[3,]} & 0.1896104 & 0.3941423 & 0.0000000 & 0.2939271\end{array}\right.$

$[4] \quad 0.1896104 \quad 0.3941423 \quad 0.4728997 \quad$,

,$\quad 2$

$$
[, 1] \quad[, 2] \quad[, 3] \quad[, 4]
$$

$\left[\begin{array}{llllllll}{[1,]} & 0.0000000 & 0.1949791 & 0.3089431 & 0.1959514\end{array}\right.$

$[2] \quad 0.1073593 \quad 0.0000000 \quad$,

$\left[\begin{array}{lllllll}{[3,]} & 0.1073593 & 0.1949791 & 0.0000000 & 0.1959514\end{array}\right.$

$\left[\begin{array}{llllllll}4,] & 0.1073593 & 0.1949791 & 0.3089431 & 0.0000000\end{array}\right.$

- q: Array of size $S \times S \times K \max$ with estimated semi-Markov kernel estSeqNP3\$q[, , 1:3]

, 1
$[, 1]$
$[, 2]$
[, 3]
$[, 4]$

$\begin{array}{llllllll}{[1,]} & 0.00000000 & 0.07792208 & 0.2562771 & 0.06753247\end{array}$

$\left[\begin{array}{lllllll}{[2,]} & 0.03508772 & 0.00000000 & 0.1378446 & 0.15956558\end{array}\right.$

$\left[\begin{array}{llllllll}{[3,]} & 0.05559322 & 0.18576271 & 0.0000000 & 0.06372881\end{array}\right.$

$\left[\begin{array}{lllllll}4,] & 0.07698541 & 0.08670989 & 0.1920583 & 0.00000000\end{array}\right.$

,$\quad 2$
$[, 1]$
[, 2]
[, 3]
$[, 4]$

$[1] \quad 0.00000000 \quad$,

$[2] \quad 0.01670844 \quad 0.00000000 \quad$,

$[3] \quad 0.03796610 \quad 0.09762712 \quad 0.0000000 \quad$,

$[4] \quad 0.038897890 .031604540 .1385737 \quad$,

,$\quad 3$

$$
[, 1] \quad[, 2] \quad[, 3] \quad[, 4]
$$

$\left[\begin{array}{lllllll}{[1,]} & 0.00000000 & 0.02770563 & 0.07965368 & 0.04069264\end{array}\right.$

$[2] \quad 0.07101086 \quad$,

$[3] \quad 0.09152542 \quad 0.054237290 .00000000 \quad$,

$\left[\begin{array}{lllllllll}4,] & 0.10940032 & 0.01782820 & 0.05591572 & 0.00000000\end{array}\right.$ 


\section{Supplementary functions}

In this package, others functions are available. These functions enable to compute the initial distribution for a semi-Markov model, the log-likelihood of a semi-Markov model and the AIC and BIC of a semi-Markov model.

InitialLawSM(): Estimation of initial distribution for a semi-Markov model

\section{Parameters:}

- q: Array of size $S \times S \times K \max$ with estimated semi-Markov kernel

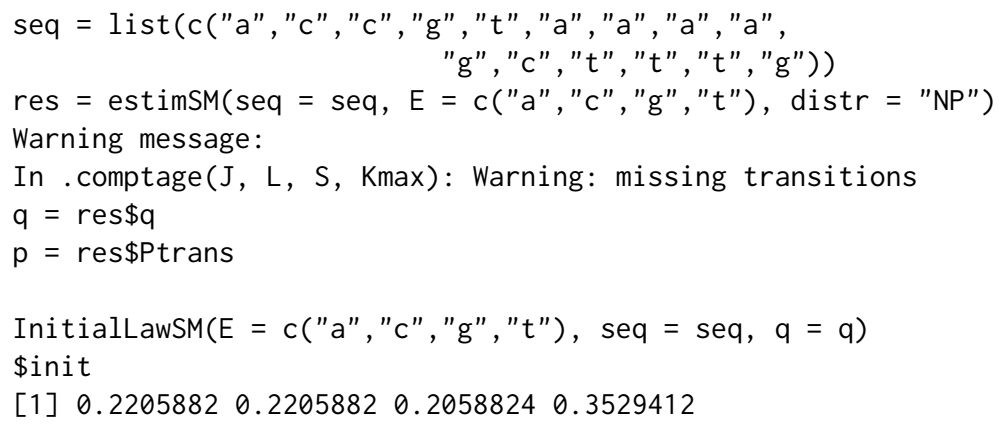

Values: The function InitialLawSM() returns a list containing a vector of the initial distribution.

LoglikelihoodSM (): Computation of the log-likelihood

\section{Parameters:}

- E: Vector of state space of length $S$

- seq: List of the sequence(s) from which to estimate

- mu: Vector of initial distribution of length $S$

- Ptrans: Matrix of transition probabilities of the embedded Markov chain $J=\left(J_{m}\right)_{m}$ of size $S \times S$

- TypeSojournTime: Type of sojourn time; it can be "fij", "fi", "fj" or "f" according to the four cases previously discussed

- distr: Sojourn time distributions:

- is a matrix of distributions of size $S \times S$ if TypeSojournTime is equal to " $f i j "$,

- is a vector of distributions of size $S$ if TypeSojournTime is equal to " $f i$ " or " $f j$ ",

- is a distribution if TypeSojournTime is equal to " $f$ ",

where the distributions to be used can be one of "uniform", "geom", "pois", "weibull" or "nbinom".

- param: Parameters of sojourn time distributions:

- is an array of parameters of size $S \times S \times 2$ if TypeSojournTime is equal to " $\mathrm{fij}$ "

- is a matrix of parameters of size $S \times 2$ if TypeSojournTime is equal to " $f i$ " or " $f j$ "

- is a vector of parameters if TypeSojournTime is equal to " $f$ "

- laws: Sojourn time distributions introduced by the user:

- is an array of size $S \times S \times K \max$ if TypeSojournTime is equal to " $\mathrm{fij}$ ",

- is a matrix of size $S \times K \max$ if TypeSojournTime is equal to " $\mathrm{fi}$ " or " $\mathrm{fj}$ ",

- is a vector of length Kmax if TypeSojournTime is equal to " $f$ ",

where Kmax is the maximum length for the sojourn times. 
\#\# state space

$E=c(" a ", " c ", " g ", " t ")$

$\mathrm{S}=\operatorname{length}(\mathrm{E})$

\#\# creation of transition matrix

$\operatorname{Pij}=\operatorname{matrix}(\mathrm{c}(0,0.2,0.3,0.4,0.2,0,0.5,0.2,0.5,0.3,0,0.4,0.3,0.5,0.2,0), \mathrm{ncol}=4)$

\#\# for the reproducibility of the results

set. seed(3)

\#\# simulation

seq5000 $=\operatorname{simulSM}(E=E$, NbSeq $=1$, lengthSeq $=5000$,

TypeSojournTime $=$ " $f$ ", init $=c(1 / 4,1 / 4,1 / 4,1 / 4)$,

Ptrans $=$ Pij, distr $=$ "pois", param = 2, File.out = "seq5000.txt")

\#\# computation of the log-likelihood

$\operatorname{LoglikelihoodSM}($ seq $=\operatorname{seq5000}, E=E, m u=\operatorname{rep}(1 / 4,4)$,

Ptrans $=$ Pij, distr $=$ "pois", param $=2$,

$\$ L$

TypeSojournTime $=$ "f")

$\$ L[[1]]$

[1] -1748.431

$\$$ Kmax

[1] 10

Values: The function likelihoodSM() returns a list containing:

- L: List with the value of the likelihood for each sequence

- Kmax: Maximal sojourn time

We also consider model selection criteria in order to evaluate and choose among candidate models; the Akaike information criterion (AIC) and the Bayesian information criterion (BIC) are considered.

AIC_SM(): computation of the AIC

$$
\operatorname{AIC}(M)=-2 \log \mathcal{L}+2 M,
$$

where $\mathcal{L}$ is the log-likelihood, $M$ is the number of parameters involved in the model

\section{Parameters:}

- E: Vector of state space of length $S$

- seq: List of the sequence(s) from which to estimate

- mu: Vector of initial distribution of length $S$

- Ptrans: Matrix of transition probabilities of the embedded Markov chain $J=\left(J_{m}\right)_{m}$ of size $S \times S$

- TypeSojournTime: Type of sojourn time; it can be "fij", "fi", "fj" or "f" according to the four cases previously discussed

- distr: Sojourn time distributions:

- is a matrix of distributions of size $S \times S$ if TypeSojournTime is equal to " $f i j "$,

- is a vector of distributions of size $S$ if TypeSojournTime is equal to " $f i$ " or " $f j$ ",

- is a distribution if TypeSojournTime is equal to " $f$ ",

where the distributions to be used can be one of "uniform", "geom", "pois", "weibull" or "nbinom".

- param: Parameters of sojourn time distributions:

- is an array of parameters of size $S \times S \times 2$ if TypeSojournTime is equal to " $\mathrm{i} j$ "

- is a matrix of parameters of size $S \times 2$ if TypeSojournTime is equal to " $f \mathrm{i}$ " or " $\mathrm{fj}$ "

- is a vector of parameters if TypeSojournTime is equal to " $f$ "

- laws: Sojourn time distributions introduced by the user:

- is an array of size $S \times S \times K \max$ if TypeSojournTime is equal to " $\mathrm{fij}$ ",

- is a matrix of size $S \times K \max$ if TypeSojournTime is equal to " $\mathrm{fi}$ " or " $\mathrm{f}$ ", 
- is a vector of length Kmax if TypeSojournTime is equal to " $f$ ",

where Kmax is the maximum length for the sojourn times.

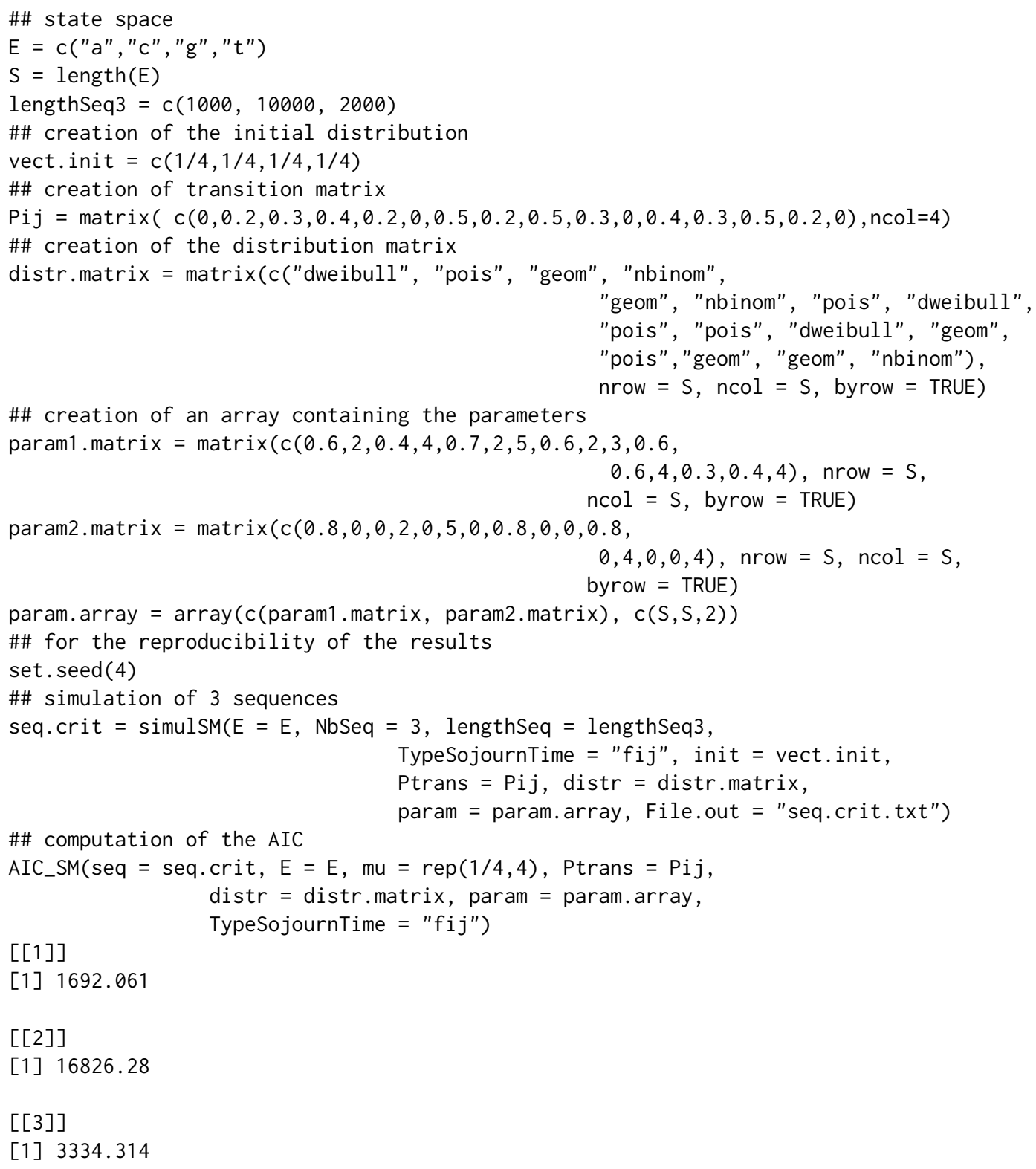

Values: The function AIC_SM() returns a list with the value of AIC for each sequence.

- BIC_SM(): computation of the BIC

$$
B I C(M)=-2 \log \mathcal{L}+\log (n) M
$$

where $\mathcal{L}$ is the log-likelihood, $M$ is the number of parameters involved in the model and $n$ is the sample size.

\section{Parameters:}

- E: Vector of state space of length $S$

- seq: List of the sequence(s) from which to estimate

- mu: Vector of initial distribution of length $S$

- Ptrans: Matrix of transition probabilities of the embedded Markov chain $J=\left(J_{m}\right)_{m}$ of size $S \times S$

- TypeSojournTime: Type of sojourn time; it can be "fij", "fi", "fj" or "f" according to the four cases previously discussed 
- distr: Sojourn time distributions:

- is a matrix of distributions of size $S \times S$ if TypeSojournTime is equal to " $f i j "$,

- is a vector of distributions of size $S$ if TypeSojournTime is equal to " $f i$ " or " $f j$ ",

- is a distribution if TypeSojournTime is equal to " $f$ ",

where the distributions to be used can be one of "uniform", "geom", "pois", "weibull" or "nbinom".

- param: Parameters of sojourn time distributions:

- is an array of parameters of size $S \times S \times 2$ if TypeSojournTime is equal to " $\mathrm{fij}$ "

- is a matrix of parameters of size $S \times 2$ if TypeSojournTime is equal to " $f i$ " or " $f j$ "

- is a vector of parameters if TypeSojournTime is equal to " $f$ "

- laws: Sojourn time distributions introduced by the user:

- is an array of size $S \times S \times K \max$ if TypeSojournTime is equal to " $\mathrm{fij}$ ",

- is a matrix of size $S \times K \max$ if TypeSojournTime is equal to " $\mathrm{f}$ " or " $\mathrm{f}$ ",

- is a vector of length Kmax if TypeSojournTime is equal to " $f$ ",

where Kmax is the maximum length for the sojourn times.

\#\# computation of the BIC

BIC_SM(seq $=$ seq3, E $=E, m u=\operatorname{rep}(1 / 4,4)$, Ptrans $=$ Pij, distr = distr.matrix, param = param.array,

$[[1]]$ TypeSojournTime $=$ "fij")

[1] 1760.811

$[[2]]$

[1] 16927.22

$[[3]]$

[1] 3412.733

Values: The function BIC_SM() returns a list with the value of BIC for each sequence.

\section{The Markov case}

In the SMM R package, we have also implemented the estimation and the simulation of discrete-time multi-state Markov models. As in the semi-Markov case, other functions are available, enabling to estimate the initial distribution, to compute the log-likelihood and also the AIC and BIC of a Markov model.

simulMk(): Simulation of a Markov chain of order $k$

\#\# state space

$E<-c(" a ", " c ", " g ", " t ")$

$S=$ length $(E)$

vect.init $<-c(1 / 4,1 / 4,1 / 4,1 / 4)$

$k<-2$

$\mathrm{p}<-\operatorname{matrix}\left(0.25\right.$, nrow $=\mathrm{S}^{\wedge} \mathrm{k}$, ncol $\left.=\mathrm{S}\right)$

\#\# for the reproducibility of the results

set.seed(5)

\#\# simulation of 3 sequences with the simulMk function

seq. markov $=\operatorname{simulMk}(E=E, n b S e q=3$, lengthSeq $=c(1000,10000,2000)$,

Ptrans $=p$, init $=$ vect.init, $k=2$, File.out $=$ "seq.markov.txt")

seq.markov[[1]][1:25]

[1] "g" "g" "g" "g" "c" "c" "c" "a" "a" "a" "c" "c" "c" "g" "g" "c" "c" "c" "c"

"c" "g" "g" "g" "a" "a"

estimMk(): Estimation of a Markov chain of order $k$ 
\#\# state space

E <- c("a", "c", "g", "t")

\#\# for the reproducibility of the results

seq. markov = read. fasta ("seq. markov.txt")

\#\# estimation of simulated sequences

res. markov $=$ estimMk (seq $=$ seq. markov, $E=E, k=2$ )

Values: The function estimMk () returns a list containing:

- init: Vector of initial probabilities of the Markov chain

res.markov\$init

$\begin{array}{lllllll} & 0.2513810 & 0.2491905 & 0.2519048 & 0.2475238\end{array}$

- Ptrans: Matrix of transition probabilities of the Markov chain

res.markov\$Ptrans

$[, 1] \quad[, 2] \quad[, 3] \quad[, 4]$

$\left[\begin{array}{lllllll}{[1,]} & 0.2845283 & 0.2498113 & 0.2188679 & 0.2467925\end{array}\right.$

$\begin{array}{llllllll}{[2,]} & 0.2479645 & 0.2361214 & 0.2709104 & 0.2450037\end{array}$

$\begin{array}{lllllll}{[3,]} & 0.2640625 & 0.2507813 & 0.2515625 & 0.2335937\end{array}$

$\left[\begin{array}{llllllll}4,] & 0.2577475 & 0.2448980 & 0.2433862 & 0.2539683\end{array}\right.$

$\begin{array}{lllllllll}{[5,]} & 0.2371988 & 0.2454819 & 0.2402108 & 0.2771084\end{array}$

$\left[\begin{array}{lllllll}6,] & 0.2458629 & 0.2584712 & 0.2450749 & 0.2513790\end{array}\right.$

$\left[\begin{array}{lllllll}7,] & 0.2435897 & 0.2533937 & 0.2624434 & 0.2420814\end{array}\right.$

$\begin{array}{lllllllll}{[8,]} & 0.2457887 & 0.2258806 & 0.2710567 & 0.2572741\end{array}$

$\begin{array}{llllllll}{[9,]} & 0.2300296 & 0.2692308 & 0.2684911 & 0.2322485\end{array}$

$\left[\begin{array}{lllllll}{[10,]} & 0.2767584 & 0.2194190 & 0.2484709 & 0.2553517\end{array}\right.$

$\begin{array}{llllllll}{[11,]} & 0.2542248 & 0.2578986 & 0.2549596 & 0.2329170\end{array}$

$\begin{array}{llllllll}{[12,]} & 0.2377567 & 0.2535545 & 0.2440758 & 0.2646130\end{array}$

$\left[\begin{array}{lllllll}13,] & 0.2527473 & 0.2590267 & 0.2417582 & 0.2464678\end{array}\right.$

$\begin{array}{llllllll}{[14,]} & 0.2448196 & 0.2578665 & 0.2509593 & 0.2463546\end{array}$

$\begin{array}{llllllll}{[15,]} & 0.2617602 & 0.2276176 & 0.2594841 & 0.2511381\end{array}$

$\begin{array}{llllllll}{[16,]} & 0.2390469 & 0.2790161 & 0.2559570 & 0.2259800\end{array}$

- InitialLawMk(): Estimation of the initial distribution of a Markov chain of order $k$

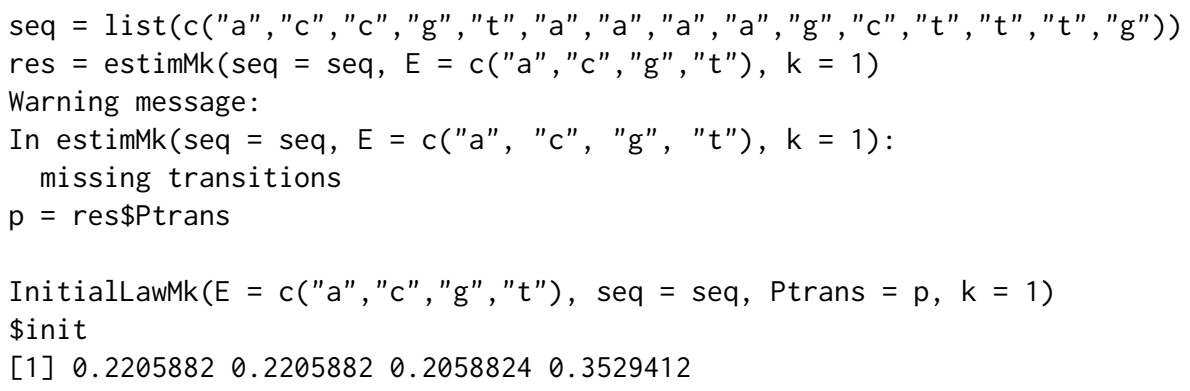

LoglikelihoodMk(): Computation of the log-likelihood

\section{Parameters:}

- mu: Initial distribution

- Ptrans: Probability transition matrix

- $\mathrm{k}$ : Order of the Markov chain

\#\# state space

$E=c(" a ", " c ", " g ", " t ")$

$S=$ length $(E)$

\#\# creation of transition matrix

$\mathrm{p}=\operatorname{matrix}(\operatorname{rep}(1 / 4, S * S), \mathrm{ncol}=4)$

\#\# for the reproducibility of the results

set.seed(6)

\#\# simulation of two sequences of length 20 and 50 respectively

seq. markov2 $=\operatorname{simulMk}(E=E, n b S e q=2$, lengthSeq $=c(20,50)$, 


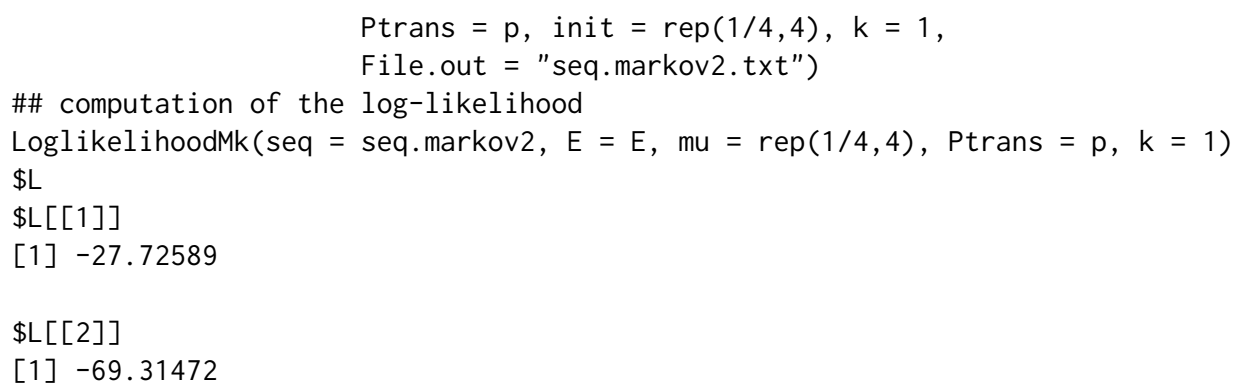

Values: The function likelihoodSM() returns a list containing the value of the likelihood for each sequence.

aIC_Mk(): Computation of the AIC for a Markov chain of order $k$

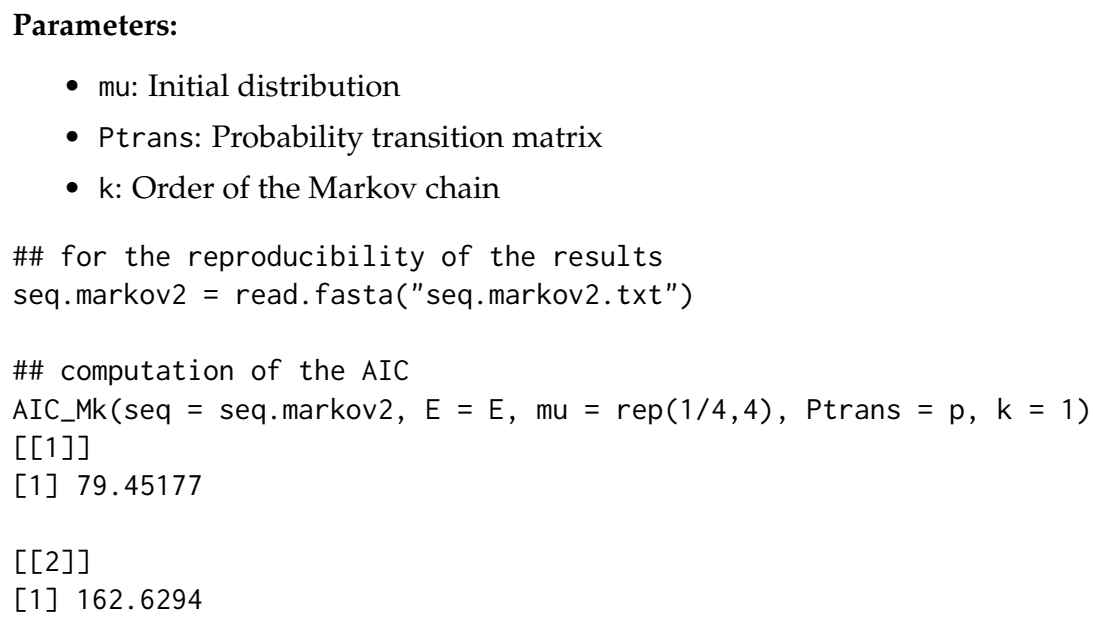

Values: The function AIC_Mk() returns a list containing the value of the AIC for each sequence.

BIC_Mk(): Computation of the BIC for a Markov chain of order $k$

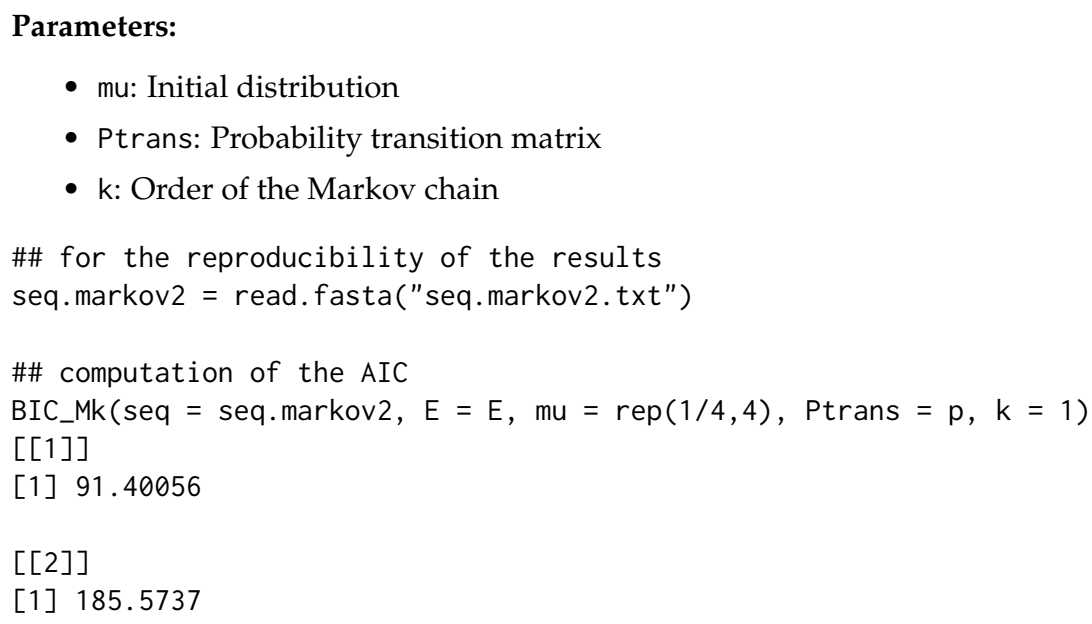

Values: The function BIC_Mk() returns a list containing the value of the BIC for each sequence.

\section{Concluding remarks}

In this paper we have presented the SMM, an R package for the simulation and the estimation of discrete-time multi-state semi-Markov models. The conditional sojourn time can be modeled by an arbitrary distribution for a semi-Markov model, which enables a generalization with respect to Markov 
models, where the sojourn time is only modelled by a Geometric distribution (in discrete time) or an Exponential distribution (in continuous time). The SMM package offers a variety of conditional sojourn time distributions (Poisson, Uniform, Negative Binomial, Geometric and Discrete Weibull). This package provides also non-parametric estimation and simulation and takes into account censored data of several types.

To summarize, the importance and interest of the R package SMM that we have developed come from:

- considering versatile tools, namely discrete-time multi-state semi-Markov processes, that are of use in a variety of applied fields, like survival analysis, biology, reliability, DNA analysis, insurance and finance, earthquake modeling, meteorology studies, etc. An example of application can be the description of DNA sequences by using a 2-state process to distinguish between coding and noncoding regions in DNA sequences. It is well known that the length of such regions generally does not follow a Geometric distribution. Thus semi-Markov chains can represent adapted tools for this type of modeling.

- implementing parametric and non-parametric estimation/simulation;

- considering several censoring schemes, that important in various applications;

- taking into account one or several independent sample paths;

- considering different types of semi-Markov kernels: either of the general type $q_{i j}(k)=p_{i j} f_{i j}(k)$ with the holding time distributions $f_{i j}(k)$ depending on the current state and on the next state to be visited, or with the holding time distributions depending only on the current state, $q_{i j}(k):=p_{i j} f_{i \bullet}(k)$, or with the holding time distributions depending only on the next state to be visited, $q_{i j}(k):=p_{i j} f_{\bullet j}(k)$, or with the holding time distributions depending neither on the current, nor on the future state, $q_{i j}(k):=p_{i j} f(k)$. As already mentioned, it is important that these four types of models be considered separately.

In conclusion, the R package SMM that we have developed deals with an important and versatile tool, useful for researchers, practitioners and engineers in various fields.

\section{Bibliography}

V. S. Barbu and N. Limnios. Empirical estimation for discrete time semi-Markov processes with applications in reliability. Journal of Nonparametric Statistics, 18(4):483-498, 2006. URL https: //doi.org/10.1080/10485250701261913. [p236]

V. S. Barbu and N. Limnios. Semi-Markov Chains and Hidden Semi-Markov Models toward Applications, volume 191 of Lecture Notes in Statistics. Springer-Verlag, New York, NY, 2008. ISBN 978-0-38773171-1 978-0-387-73173-5. URL http://link. springer. com/10.1007/978-0-387-73173-5. [p226, 236]

V. S. Barbu, A. Karagrigoriou, and A. Makrides. Semi-Markov modelling for multi-state systems. Methodology and Computing in Applied Probability, 2016. ISSN 1573-7713. URL https://doi .org/10. $1007 / \mathrm{s} 11009-016-9510-y$. [p226]

J. Bulla and I. Bulla. Stylized facts of financial time series and hidden semi-Markov models. Comput. Statist. Data Anal., 51:2192-2209, 2006. URL https://doi .org/10.1016/j . csda. 2006.07.021. [p226]

J. Bulla, I. Bulla, and O. Nenadić. Hsmm - An R package for analyzing hidden semi-Markov models. Computational Statistics \& Data Analysis, 54(3):611-619, 2010. ISSN 0167-9473. URL https://doi. org/10.1016/j.csda.2008.08.025. [p226]

O. Chryssaphinou, M. Karaliopoulou, and N. Limnios. On discrete time semi-Markov chains and applications in words occurrences. Comm. Statist. Theory Methods, 37:1306-1322, 2008. URL https: //doi.org/10.1080/03610920701713328. [p226]

G. D'Amico, F. Petroni, and F. Prattico. Wind speed modeled as an indexed semi-Markov process. Environmetrics, 24(6):367-376, 2013. ISSN 1099-095X. URL https://doi .org/10. 1002/env. 2215. [p226] 
G. D'Amico, J. Janssen, and R. Manca. Downward migration credit risk problem: a non-homogeneous backward semi-Markov reliability approach. Journal of the Operational Research Society, 67(3):393-401, 2016a. ISSN 1476-9360. URL https://doi.org/10.1057/jors. 2015.35. [p226]

G. D'Amico, R. Manca, C. Corini, F. Petroni, and F. Prattico. Tornadoes and related damage costs: Statistical modelling with a semi-Markov approach. Geomatics, Natural Hazards and Risk, 7(5): 1600-1609, 2016b. URL https://doi .org/10.1080/19475705.2015.1124462. [p226]

N. Heutte and C. Huber-Carol. Semi-Markov models for quality of life data with censoring. In M. Mesbah, M.-L. T. Lee, and B. F. Cole, editors, Statistical Methods for Quality of Life Studies, pages 207-218. Kluwer, Dordrecht, 2002. URL https://doi .org/10.1007/978-1-4757-3625-0_16. [p226]

J. Janssen and R. Manca. Applied Semi-Markov Processes. Springer-Verlag, 2006. URL https: //doi .org/ 10.1007/0-387-29548-8. [p226]

A. Król and P. Saint-Pierre. SemiMarkov : An $R$ Package for Parametric Estimation in Multi-State Semi-Markov Models. Journal of Statistical Software, 66(6), 2015. ISSN 1548-7660. URL https: //doi.org/10.18637/jss.v066.i06. [p226]

P. Lévy. Processus semi-markoviens. In Proc. of International Congress of Mathematics, Amsterdam, 1954. [p226]

N. Limnios and G. Oprisan. Semi-Markov Processes and Reliability. Birkhäuser, Boston, 2001. URL https://doi.org/10.1007/978-1-4612-0161-8. [p226, 237]

J. O'Connell and S. Højsgaard. Hidden Semi Markov Models for Multiple Observation Sequences: The mhsmm Package for R. Journal of Statistical Software, 39(4), 2011. ISSN 1548-7660. URL https://doi.org/10.18637/jss.v039.i04. [p226]

B. Ouhbi and N. Limnios. Nonparametric reliability estimation for semi-Markov processes. J. Statist. Plann. Inference, 109(1-2):155-165, 2003. URL https://doi .org/10. 1016/s0378-3758(02)00308-7. [p226]

J. Sansom and P. J. Thomson. Fitting hidden semi-Markov models to breakpoint rainfall data. J. Appl. Probab., 38A:142-157, 2001. URL https://doi .org/10.1239/jap/1085496598. [p226]

W. L. Smith. Regenerative stochastic processes. Proc. R. Soc. Lond. Ser. A Math. Phys. Eng., 232:6-31, 1955. URL https://doi .org/10.1098/rspa.1955.0198. [p226]

L. Takacs. Some investigations concerning recurrent stochastic processes of a certain type. Magyar Tud. Akad. Mat. Kutato Int. Kzl., 3:115-128, 1954. [p226]

S. Trevezas and N. Limnios. Exact MLE and asymptotic properties for nonparametric semi-Markov models. Journal of Nonparametric Statistics, 23(3):719-739, 2011. URL https://doi .org/10.1080/ 10485252.2011.555543. [p236, 237]

I. Votsi, N. Limnios, G. Tsaklidis, and E. Papadimitriou. Estimation of the expected number of earthquake occurrences based on semi-Markov models. Methodology and Computing in Applied Probability, 14(3):685-703, 2012. ISSN 1573-7713. URL https://doi.org/10.1007/s11009-0119257-4. [p226]

I. Votsi, N. Limnios, G. Tsaklidis, and E. Papadimitriou. Hidden semi-Markov modeling for the estimation of earthquake occurrence rates. Communications in Statistics: Theory and Methods, 43: 1484-1502, 2014. URL https://doi .org/10.1080/03610926.2013.857414. [p226]

Vlad Stefan Barbu

Université de Rouen-Normandie

Laboratoire de Mathématiques Raphaël Salem

UFR des Sciences et Techniques

Avenue de l'Université, BP. 12

76801 Saint-Étienne-du-Rouvray, France

E-mail: barbu@univ-rouen.fr

URL: http://Imrs. univ-rouen. fr/persopage/barbu

Caroline Bérard

Université de Rouen-Normandie 
LITIS EA 4108

E-mail: caroline. berard@univ-rouen.fr

Dominique Cellier

Université de Rouen-Normandie

LITIS EA 4108

E-mail: dominique.cellier@laposte.net

Mathilde Sautreuil

Université de Rouen-Normandie

Laboratoire de Mathématiques Raphaël Salem

UFR des Sciences et Techniques

Avenue de l'Université, BP. 12

76801 Saint-Étienne-du-Rouvray, France

E-mail: mathilde.sautreuilegmail.com

Nicolas Vergne

Université de Rouen-Normandie

Laboratoire de Mathématiques Raphaël Salem

UFR des Sciences et Techniques

Avenue de l'Université, BP. 12

76801 Saint-Étienne-du-Rouvray, France

E-mail: nicolas.vergne@univ-rouen. fr

URL: http://Imrs. univ-rouen.fr/persopage/nicolas-vergne 